\title{
Fork head transcription factor is required for ovarian mature in the brown planthopper, Nilaparvata lugens (Stål)
}

\author{
Xiaolin Dong, Yifan Zhai, Jianqing Zhang, Zhongxiang Sun, Jing Chen, Jie Chen and Wenqing Zhang*
}

\begin{abstract}
Background: The brown planthopper (BPH), Nilaparvata lugens, is the most devastating rice pest in many areas throughout Asia. The reproductive system of female N. lugens consists of a pair of ovaries with 24-33 ovarioles per ovary in most individuals which determine its fecundity. The fork head (Fox) is a transcriptional regulatory molecule, which regulates and controls many physiological processes in eukaryotes. The Fox family has several subclasses and members, and several Fox factors have been reported to be involved in regulating fecundity.

Results: We have cloned a fork head gene in N. lugens. The full-length cDNA of NIFoxA is 1789 bp and has an open reading frame of $1143 \mathrm{bp}$, encoding a protein of 380 amino acids. Quantitative real-time PCR (RT-qPCR) and Reverse Transcription- PCR (RT-PCR) analysis revealed that NIFoxA mRNA was mainly expressed in the fat body, midgut, cuticle and Malpighian tube, and was expressed continuously with little change during all the developmental stages. N/FoxA belongs to the FoxA subfamily of the Fox transcription factors. Knockdown of NIFoxA expression by RNAi using artificial diet containing double-stranded RNA (dsRNA) significantly decreased the number of offspring and impacted the development of ovaries. ELISA and Western blot analyses showed that feeding-based RNAi of NIFoxA gene also resulted in decreased expression of vitellogenin ( $\mathrm{Vg}$ ) protein.

Conclusion: N/FoxA plays an important role in regulation of fecundity and development of ovaries in the BPH via regulating vitellogenin expression.
\end{abstract}

\section{Background}

The brown planthopper (BPH), Nilaparvata lugens, is a major pest to rice production in many areas of Asia. It shows two wing forms, long (macropterous) and short (brachypterous) ones, in its adult stage. The macropterous adults possess the ability to migrate across long distances, while the brachypterous adults exemplify strong reproductive capacities. One individual brachypterous female has the ability to produce a large number of offspring, increasing each generation size by 10 - to 40 folds $[1,2]$. The macropterous adults migrate long distances every year to the rice-growing areas of China, Japan and Korea, resulting in severe infestations $[3,4]$, causing hopperburn and ultimately leading to reductions in rice yields. The $\mathrm{BPH}$ ingests nutriments specifically from the phloem of rice plants with its stylet, causing

* Correspondence: wenqingzhang818@yahoo.com.cn

School of Life Sciences, Sun Yat-sen University, Guangzhou, Guangdong, 510275 China whole plants to become yellow and rapidly dry, a phenomenon referred to as hopperburn [5]. In additional, $\mathrm{BPH}$ is a vector for some rice diseases such as the rice ragged stunt virus (RRSV) and rice grassy stunt virus (RGSV) [6]. BPH often causes losses of up to $60 \%$ in rice crop yields [7]. In China, N. lugens covered areas totaling 9.3 million $\mathrm{hm}^{2}$ in 1974, and in 2005, the affected area has increased to 23.23 million $\mathrm{hm}^{2}$, causing huge economic losses [8]. Because insecticides have been extensively used to control this pest, resistances have begun to arise in different countries and areas [9-11]. This pattern of resistance causes ecological imbalances in predator-prey relationships and has often resulted in the resurgence of $\mathrm{BPH}[12]$. Despite the extensive literature documenting the effects of host plant quality on the performance of herbivorous insects, surprisingly few publications have considered the fecundity of the BPH.

\section{Biomed Central}


The reproductive system of female insects consists of a pair of ovaries, ovarioles is the function units of the ovary containing a series of tapering egg tubes. There is a progression of developing oocytes in the ovarioles. The number of ovarioles in each ovary varies tremendously in different insect species and determines fecundity [13]. The $N$. lugens has 24-33 ovarioles per ovary in most individuals [14].

The fork head (Fox) family transcription factors have several subclasses and members, designated as A to $Q$ $[15,16]$, and they share a structurally conservative fork head box defined by a 'winged helix' DNA-binding domain [16]. The first fork head protein, FoxA, was first found in D. melanogaster and importantly required in the embryonic development [17]. Subsequently, several additional Fox transcription factors have been described in various organisms [18]. It has been reported that Fox transcription factors play critical roles in regulation of metabolism, proliferation, apoptosis, development, organogenesis, differentiation and control of oxidative stress through both activation and repression of target gene expression by multiple mechanisms [18-21]. In some insects, Fox factors participate in the regulation of many physiological processes [22-24]. Reports have attested that endocrine hormones play an important role in the development of ovaries [25]. Several Fox factors have been shown to be involved in regulating vitellogenesis, fecundity and ovarian events such as follicular development and selection, ovarian cell proliferation and cancerogenesis, ovarian cell apoptosis, ovarian secretory activity and oocyte/cumulus maturation [26,27]. However, whether FoxA influences reproduction or not is still unknown.

RNA interference (RNAi) through double-stranded RNA (dsRNA) has been used widely to study gene function in insects. Since RNAi was first discovered in the nematode Caenorhabditis elegans [28]. RNAi has extensively applied in ovarian function study [29]. Many means of transporting dsRNA into the body of an organism have been explored, including microinjection, soaking, oral feeding and transgenic plant expression [30-34]. Recently, it has been reported that gene knockdown in several insects can be achieved successfully through feeding the insects bacteria that express dsRNA $[34,35]$ or artificial diet containing dsRNA [36]. Directly feeding dsRNA is a simple manipulation, inflicts no body injury and has been established for insect research.

The present study was designed to characterize and identify expression of the transcription factor FoxA in Hemiptera, N. lugens. We used RNAi technique to knockdown expression of $N l$ FoxA by feeding the insects an artificial diet containing dsRNA. We also intended to provide evidences to confirm whether FoxA influences reproduction of $N$. lugens and whether it is involved in fundamental biological phenomena and agricultural problems related to the $\mathrm{BPH}$.

\section{Results}

Isolation and characterization of NIFoxA cDNA

Based on the conserved sequence of fork head from $T$. castaneum, D. melanogaster and B. mori, a 434-bp cDNA fragment was obtained by homologous cloning using cDNA from the $2^{\text {nd }}$ day of brachypterous female adults as a template. Sequence analysis showed that the deduced amino acid sequence of the cDNA fragment had 41-45\% identity to the corresponding region of FoxA from $T$. castaneum, D. melanogaster and B. mori. Then, the 5'- and 3'RACE were performed with two pairs of specific primers designed based on the nucleotide sequence of the cDNA fragment. A full-length NlFoxA of 1789 bp (GenBank accession No: JF345255) was obtained by overlapping the RACE fragments and the cDNA fragment. Sequence analysis showed that the open reading frame (ORF) of $N l$ FoxA is $1143 \mathrm{bp}$, encoding 380 amino acid residues with a predicted MW of $42.2 \mathrm{kDa}$ and a $\mathrm{pI}$ of 8.6 , and there is a 5 untranslated region (UTR) of 244 bp and a 3'-UTR of 453 bp (Figure 1). An 18-residue signal peptide at the N-terminus was identified by SignalP, thus the mature protein (362 amino acids) has a calculated molecular mass of $40.3 \mathrm{kDa}$ and an estimated pI of 8.6. BLAST searches using $N l$ FoxA as a query showed that all the predicted FoxA proteins, including $N l$ FoxA, from insects and vertebrates contain only one Fox DNA-binding domain, indicating that $N l$ FoxA belonged to the Fox DNA-binding superfamily. $N l$ FoxA contained typical amino acids within the 110 -aalong fkh domain, suggesting that it encodes FoxA [37].

\section{Developmental and tissue specific expression of NIFoxA}

To determine whether NlFoxA is present during developmental stages and in various tissues in the $3^{\text {rd }}$ day of brachypterous female adult $N$. lugens, total RNA of each sample was isolated. We used RT-qPCR and RT-PCR to characterize the pattern of developmental expression of NlFoxA gene for all developmental stages (including nymphs from the $1^{\text {st }}$ to $5^{\text {th }}$ instars and 1-12 days brachypterous female adults). The results demonstrated that NlFoxA mRNA was expressed at constantly low levels with little change during developmental stages (Figure 2A \&2B). A high level of NlFoxA expression was detected in the fat body, midgut, cuticle and the Malpighian tube, but was not found in the ovary and thoracic muscle (Figure 3A \&3B).

\section{Efficiency analyses after ingestion of dsNIFoxA}

After feeding synchronous nymph groups on $0.5 \mu \mathrm{g} / \mu \mathrm{l}$ NlFoxA dsRNA, dsGFP and the blank control from the $3^{\text {rd }}$ to the $5^{\text {th }}$ instar (10 days), the survival rates were $78.52 \%, 82.22 \%$ and $82.96 \%$, respectively. To investigate 


\begin{abstract}
1 GCGGGGGTCAAGGCTGGTGATTGGTGCACGGGTTCAGCCCCTCCCTCAGTCGGGTGCCGMTTCAGGTGAAGCCAC

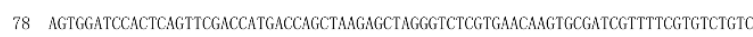

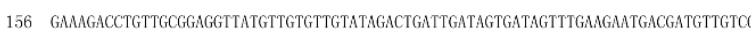

M T

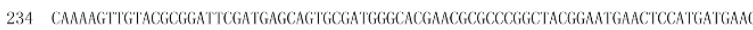
Q $\quad K \quad L \quad Y \quad A \quad D \quad S \quad M \quad S \quad S$ A M G

312 TCGTGCTCACCTCAGgGTGCCTCAgGatTCAACATGCTACAAGGCTCAGCCGCTATGGCCGgCCTCAaCgGCAACTGI $\begin{array}{cccccccccccccccccccccccccc}S & C & S & P & Q & G & A & S & G & F & N & M & L & Q & G & S & A & A & M & A & G & L & V & G & N & C\end{array}$

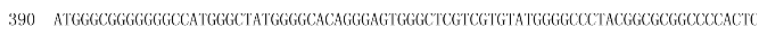
M G G G G G A M G G Y G G A Q Q G V V G G S S S C C M G G P

468 ACCTCCAGGGCGGACGTCACCCCGTCGGCGCCTCOCGGTGAAGCCGCGTCACCTTCCGCTCTGCAGAGAGCTCGTGCC

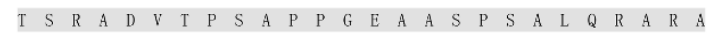

546 GACAAATCCTACCGGAGGAGTTACACGCACGCTAAACCACCCTACTCGTACATTTCGCTCATCACGATGGCCATCCAG $\begin{array}{llllllllllllllllllllllllll}D & K & S & Y & R & R & S & Y & T & H & A & K & P & P & Y & S & Y & \text { I } & S & L & \text { I } & \text { T } & M & \text { A } & \text { I } & Q\end{array}$

642 AATTCTCCCACCCGCATGCTCACACTATCAGAGACCTATCAGTTCATAATGGACCTGTTTCCGTTCTACAGGCAAAAC $\begin{array}{llllllllllllllllllllllllll}N & S & P & T & R & M & L & T & L & S & E & T & Y & Q & F & I & M & D & L & F & P & F & Y & R & Q & N\end{array}$

702 CAGCAGCGGTGGCAGAACTCGATTAGGCATTCGTTGAGTTTCATGACTGTTTTGTGAAGGTGCCGCGAACGCCGGAC Q Q $\quad$ R

780 MGCCGGGCAMGGCAGCTTCTGGACGCTGCATCCCGAGTCGGGCAACATGTTCGAGACGGCTGCTACCTGCGGAGG $\begin{array}{lllllllllllllllllllllllllll}K & P & G & K & G & S & F & W & T & L & H & P & E & S & G & N & M & F & E & N & G & C & Y & L & R & R\end{array}$

858 CAMAGCGGTTCAGGGCGAGMGMGGAGGCTGTGAGACAGACGCACAAGAGTGCGGTGAGCCCGTCGCACCACGGA $\begin{array}{lllllllllllllllllllllllllll}Q & K & R & F & K & D & E & K & K & E & A & V & R & Q & T & H & K & S & A & V & S & P & S & H & H & G\end{array}$

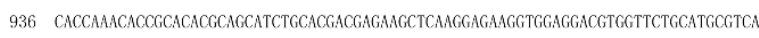

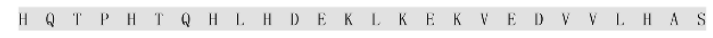

1014 CACGTTGACTGTGCTCGGCTGCCTCACAGCTGCACCAGCAGCAACAACAgtCGCATCACCAGCAACAACAACAACAG $\begin{array}{llllllllllllllllllllllllll}H & V & D & L & C & S & A & A & S & Q & L & H & Q & Q & Q & Q & Q & S & H & H & Q & Q & Q & Q & Q & Q\end{array}$

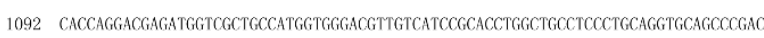
$\begin{array}{llllllllllllllllllllllllll}H & Q & D & E & M & V & A & A & M & V & G & R & C & H & P & H & L & A & A & S & L & Q & V & Q & P & D\end{array}$

1170 ACGCACTACCCTCACTCGCACCACCACCTGAAGCAGGAGTACCCCGCCTCCAGCCACCCGTTCTCCATCACCCGGCTG

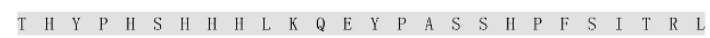

1248 CtGCCCGCCGAGAGCAAGACCGaCATCAAAATGTATGACATGTCCCAGTATGGCTACAACGACTACTACCAGAGCTCG $\begin{array}{llllllllllllllllllllllllll}L & P & A & E & S & K & T & D & I & K & M & Y & D & M & S & Q & Y & G & Y & N & D & Y & Y & Q & S & S\end{array}$

1326 CTCTACCACCCTTCGGCCGGCACCACCGCCTTGTGACAGTATCCGCTCGCCTAAGGCCATCTTAGCCCCCCCCCTCAA

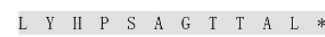

1404 GAGTTGGTGTGGCTTATAATAGTTCAAATGTGGGAGGCTACAACCAATCGCTGGGCGTAGCTCCGTGGATATCTTGAT 1482 GGACTGAGGACGAGATATGTCACAACCTCCAGGTCAGACGACAACAATCTTAGTGATATTTTTTATATAGTTTCTGAT 1560 CTCCGAATAGAMAMTCAGATAMGGCCTAATCACAMGMACACTTTGGGCCACTGGTTCAGTGCGTGGTGCGTGCA

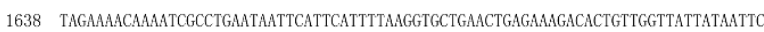

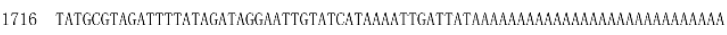

Figure 1 Nucleotide and deduced amino acid sequences of FoxA from $\boldsymbol{N}$. lugens. " "*" terminal codon; underline: predicted signal peptide.

the efficiency of RNAi after ingestion of ds NlFoxA in N. lugens, NlFoxA mRNA relative levels were measured by RT-qPCR in brachypterous adults collected 1-8 days after emergence on rice plants.

The transcript levels of NlFoxA were decreased by $55.34 \%-61.28 \%$ from the emergence of brachypterous adults up to the $8^{\text {th }}$ day of adults compared to the blank control (Figure 4). This result confirmed that RNAimediated knockdown of NlFoxA was highly effective.

\section{Knockdown of NIFoxA or NIVg reduces fecundity and disrupts ovarian development}

Once insects emerged, we successfully allocated them into 18 pairs per group. The number of offspring from

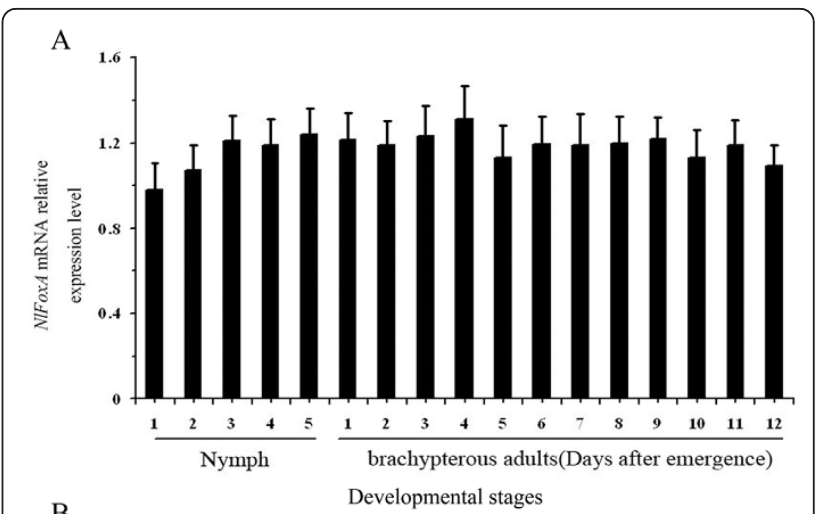

B

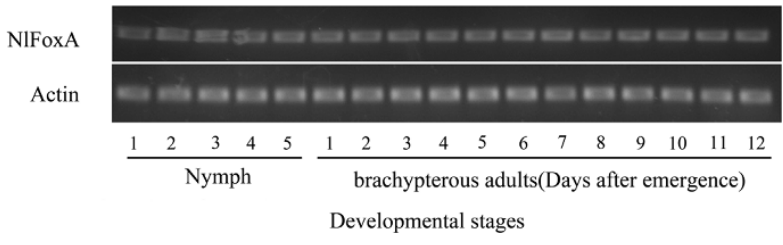

Figure 2 Expression of NIFoxA gene at different developmental stages was determined by RT-qPCR (A) and RT-PCR (B). The mRNA level was normalized relative to the $\beta$-ACTIN transcript. Each point represents mean value \pm S.E.M of three independent experiments with three individuals in each replicate.

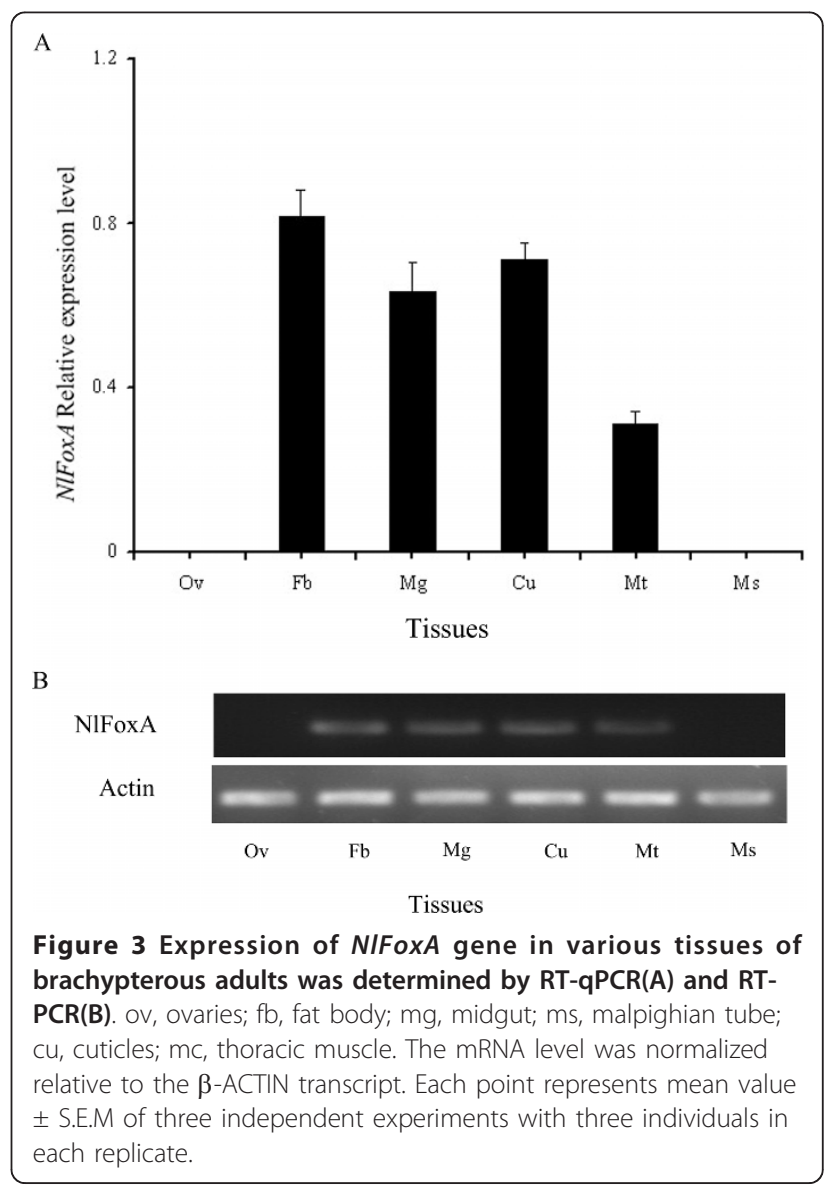




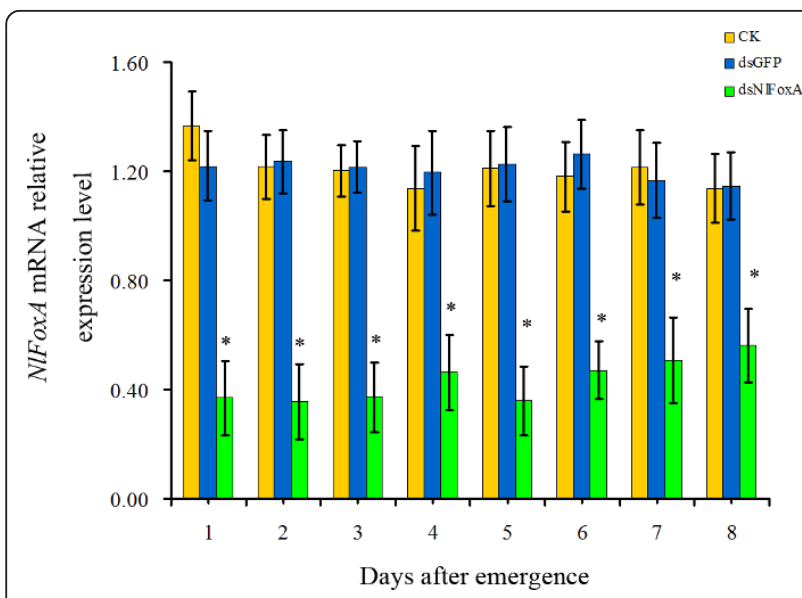

Figure 4 Detection of the efficiency of feeding-based RNAi and impact on NIFoxA mRNA level by RT-qPCR. The expression levels of $N$. lugens FoxA mRNA after different treatments. CK, control; dsGFP, $0.5 \mu \mathrm{g} / \mu \mathrm{lds}$ GFP; ds N/FoxA, $0.5 \mu \mathrm{g} / \mu \mathrm{l}$ ds NIFoxA, respectively. The data represent the mean values \pm S.E.M of three replicates. ${ }^{\prime *}$ 'means statistically significant difference in expression levels between CK and ds N/FoxA(t-test, $\mathrm{p}<0.05)$.

every individual brachypterous female adult was counted, and the result demonstrated that $N l$ FoxA plays an important role in the reproduction of $N$. lugens. The average number of offspring in the group treated with $0.5 \mu \mathrm{g} / \mu \mathrm{l} N l F o x A$ dsRNA was 104.68 , significantly less than those in the control and dsGFP-treated groups (Figure 5A). Interestingly, knockdown of $\mathrm{NlVg}$ showed similar results to those of the $N l$ FoxA knockdown (Figure 5B).

Although feeding dsNlFoxA reduced fecundity, the hatchability rate was unchanged (data not shown). Thus, we set out to determine whether ingestion of dsNlFoxA affects the development of ovaries by analysis of morphology. Assessment of ovaries from the $3^{\text {rd }}$ day of brachypterous adults suggested that RNAi knockdown of NlFoxA resulted in underdeveloped ovaries with less

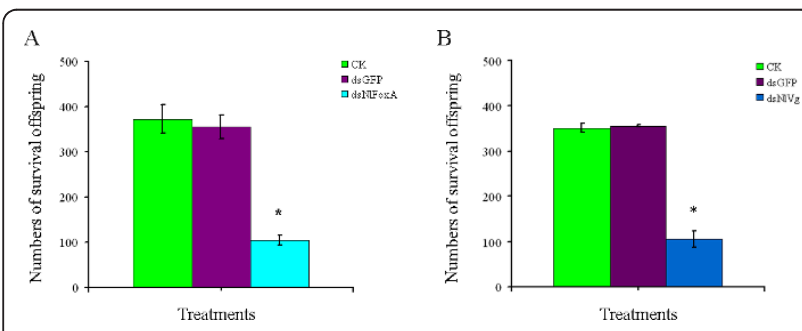

Figure 5 RNAi-mediated knockdown of NIFoxA (A) or NIVg(B) gene decrease offspring (eighteen pairs were analyzed per group). CK, control; dsGFP, $0.5 \mu \mathrm{g} / \mu \mathrm{lds}$ GFP; ds N/FoxA, $0.5 \mu \mathrm{g} / \mu \mathrm{l}$ ds NIFoxA; ds NNg, $0.5 \mu \mathrm{g} / \mu \mathrm{l}$ ds N/Vg, respectively. The data represent the mean values \pm S.E.M of three replicates. ${ }^{* \prime}$ means statistically significant difference in number of offspring between $C K$ and $d s$ NIFOXA (t-test, $\mathrm{p}<0.05)$. ovarioles and fewer eggs. In contrast, the control and dsGFP-treated groups showed repletion and no disruption of ovarioles and eggs on the same day (Figure 6). Similar results were obtained when $N l \mathrm{Vg}$ was knocked down (data not shown).

Knockdown of NIFoxA results in lower vitellogenin $(\mathrm{Vg})$ gene expression and less $\mathrm{Vg}$ protein

Vitellogenin is a conserved yolk precursor protein that is synthesized in the fat body in invertebrates, and the yolk provides nutrition to the developing oocytes for utilization during embryogenesis. We showed that expression of vitellogenin $(\mathrm{Vg})$ gene (GenBank accession No: JF345256) was significantly suppressed after ingestion of ds NlFoxA (Figure 7).

To detect Vg protein in the ovaries of BPH after different treatments, equal amounts of total proteins from three groups were coated to microtiter plates and assayed by indirect ELISA (Figure 8A) and western blotting (Figure $8 \mathrm{~B}$ ) with corresponding primary antibodies. The Vg protein concentration in ovaries of the $3^{\text {rd }}$ day of brachypterous female adults treated with $0.5 \mu \mathrm{g} / \mu \mathrm{l}$ NlFoxA dsRNA was significantly lower than that of the control and dsGFP-treated groups. The increased concentration of NlFoxA dsRNA was accompanied with a decreased concentration of $\mathrm{Vg}$ protein (data not shown). The putative $\mathrm{Vg}$ bands were considerably more intense in the control and dsGFP-treated groups than in the $N l F o x A$ dsRNA-treated group, suggesting that $N l$ FoxA regulates ovarian maturity by controlling accumulation of $N l \mathrm{Vg}$ in the ovaries.

\section{Discussion}

The brown planthopper fosters a robust ability to produce offspring. One brachypterous adult individual insect can produce more than 400 offspring [1]. This may be the primary cause of damage caused by $\mathrm{PBH}$ on rice plants in many areas of Asia. It is commonly known that the macropterous PBH migrate from Vietnam, which is considered the major source of the northbound migration to China every year [38]. Many factors can affect the fecundity of insects. Host plant quality is a critical factor for herbivorous insects [39]. Endogenous hormones and peptides are also significant components underlying successful reproduction [25,40]. In this study, we investigated how the transcription factor $N l$ FoxA regulates fecundity and ovarian development in $N$. lugens. We first cloned and characterized a cDNA sequence from the Hemiptera insect $N$. lugens encoding FoxA, which shares a high homology with the $T$. castaneum FoxA. Pattern analysis suggests that the expression level of FoxA mRNA in N. lugens was almost constant throughout the life cycle (Figure 2A \&2B), this maybe because it regulates different physiological 


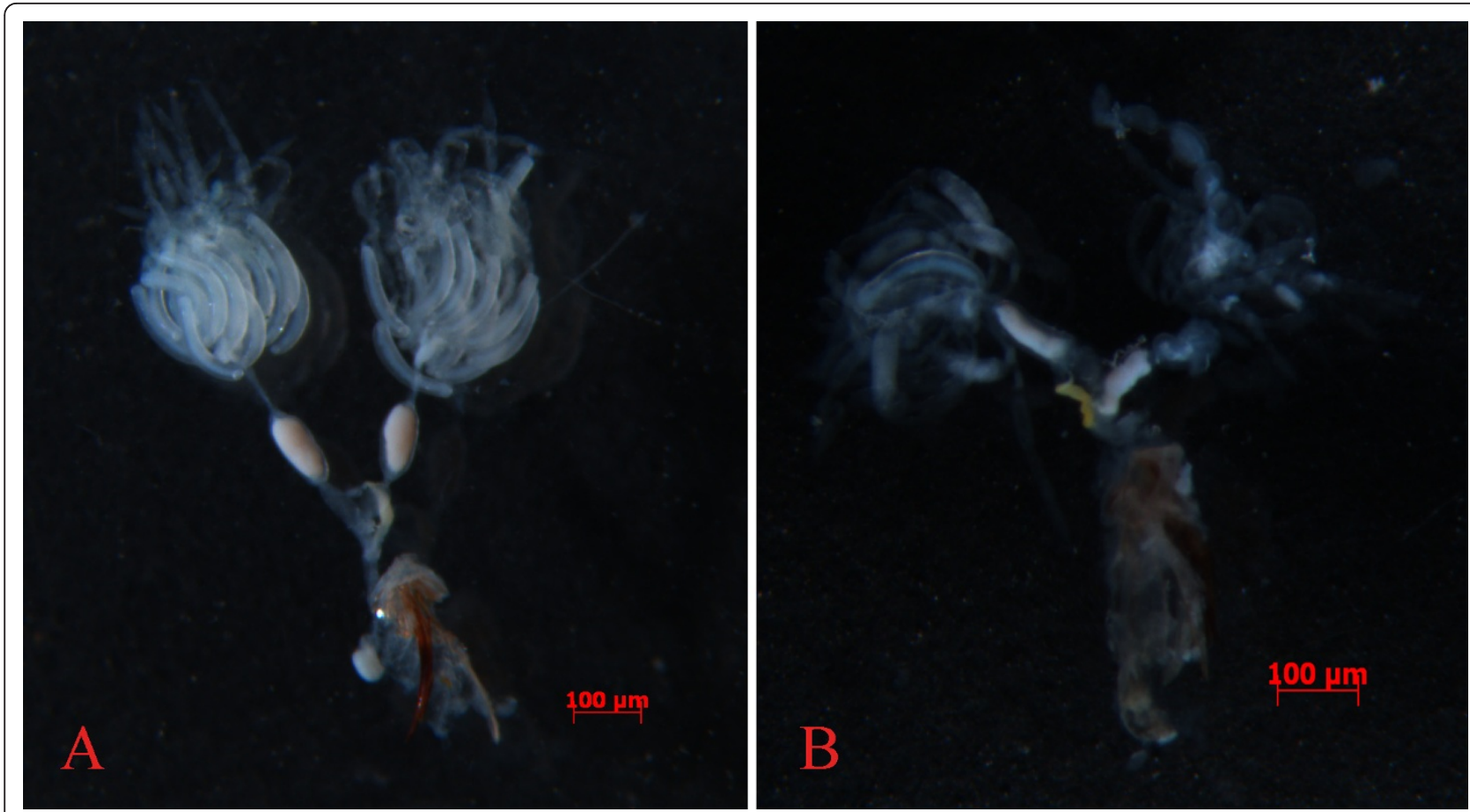

Figure 6 Ovaries dissected from the $\mathbf{3}^{\text {rd }}$ day of brachypterous female adult $\mathbf{N}$. Iugens. (A) Ovarioles from the control or $0.5 \mu \mathrm{g} / \mu \mathrm{l} \mathrm{dsGFP}$ treated insects had completely developed ripe eggs. (B) Ovaries from RNAi-treated ( $0.5 \mu \mathrm{g} / \mu \mathrm{l}$ ds NIFoxA or $0.5 \mu \mathrm{g} / \mu \mathrm{l}$ ds $\mathrm{Nl}$ Vg treated) insects with decreased number of ovarioles and fewer eggs.

processes in specific development phases. The NlFoxA was expressed in the fat body, midgut, cuticle and the Malpighian tubes, but was not found in ovaries and thoracic muscle (Figure 3A \&3B). The FoxA originates from endoderm and elongates to ectoderm during evolution [41]. In different insects, FoxA is located in different tissues. In $H$. armigera, FoxA was localized to the

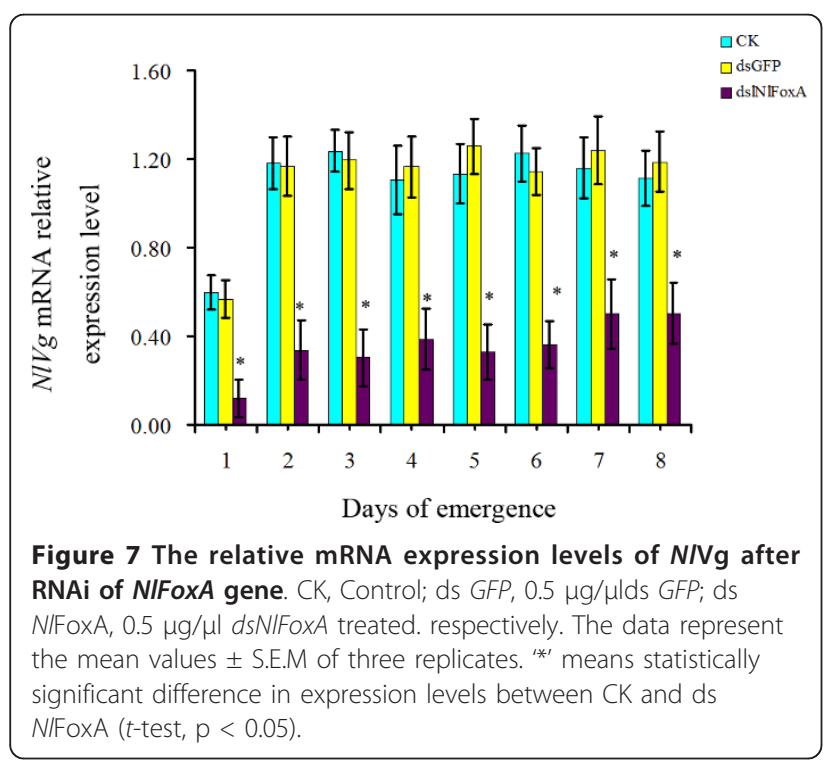

nuclei of fat body cells [23], but in Ae. Aegypti, FoxA was not expressed in fat body but in the thorax, midgut, and malpighian tubules [27]. In T. castaneum, it can be detected in yolk nuclei [37]. Our successful cloning of NlFoxA not only adds a member to this family of transcription factors but also provides a potential target for the biological control of insect pests.

Fox genes encode a family of transcription factors defined by a "winged helix" DNA-binding domain and have been identified in many metazoans, and they play critical roles in regulating diverse physiological processes. In B. mori, stabilization of SGF1/fork head to its target sequence is critical to promote fhx transcription at each intermolt [42]. In D. melanogaster, fork head controls the timing and tissue selectivity of steroidinduced developmental cell death [43]. In H. armigera, FoxA regulates the expression of diapause hormone [23]. In mammals, FoxA genes play crucial roles in multiple stages of mammalian life, including early development, organogenesis and differentiation, metabolism and homeostasis [21,44,45]. Fat body is a major tissue for vitellogenin synthesis during vitellogenesis [46]. Studies in American dog tick indicated that Vg uptake is essential for ovarian development [47]. DNA-binding assays revealed that, in Ae. aegypti, genomic DNAs containing the 5' upstream region and the positions of regulatory and coding sequences of the mosquito $\mathrm{Vg}$ gene are 


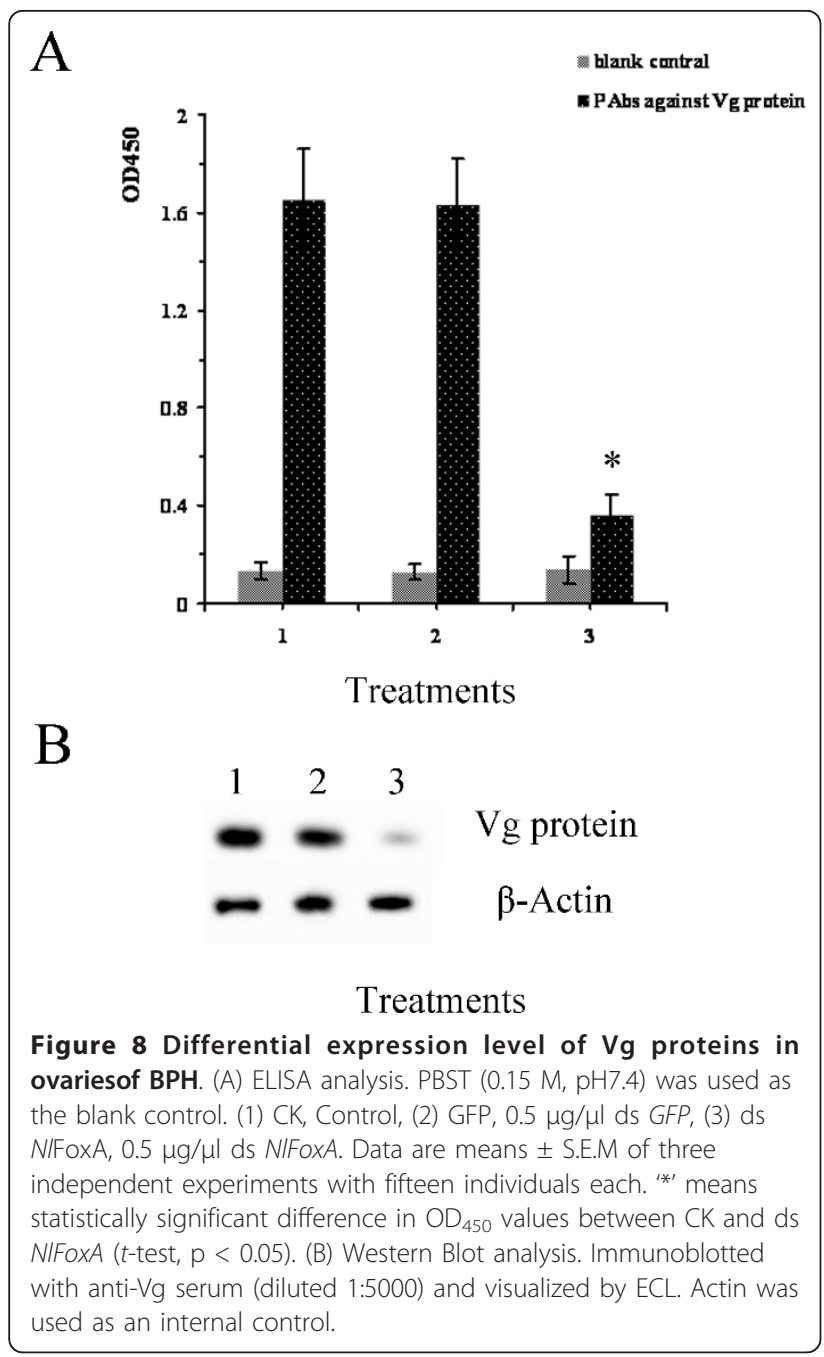

capable of binding to transcription factors [48]. Several transcription factors have been reported to be involved in Ae. aegypti fecundity, but the authors did not assess the effect on the reproduction because it is not a 'fat body' Fox factor, and the elaborate mechanisms of these phenotypes were lacking [27]. In this study, we demonstrated that NlFoxA expression level was at a constant low level with little change(Figure 2A \&2B). Interestingly, downregulation of NlFoxA can suppress ovarian development in $N$. lugens (Figure 6) and quickly decrease the number of offspring (Figure 5A). Confirming this effect, the expression levels of $N l \mathrm{Vg}$ mRNA (Figure 7) and protein (Figure 8A \&8B) were remarkably decreased. Simultaneously, knockdown of $N l \mathrm{Vg}$ had similar efficiency (data not shown). In addition, NlFoxA is also expressed in the fat body syntopogenic with $\mathrm{Vg}$ (Figure 3A \&3B). FoxA can bind to the promoters of diapause hormone and pheromone biosynthesis-activating neuropeptide to regulate their expression [23]. Whether $N l$ FoxA regulates $N l \mathrm{Vg}$ by directly binding to regulatory elements, inducing hormone synthesis via the other pathway, or supporting the nutritional element of ovary development requires further deliberation.

RNAi has been widely used to study gene function in insects. There are many approaches for delivering dsRNA into an organism's body. It has been reported that gene knockdown in several insects can be achieved successfully through feeding dsRNA. The oral delivery of an artificial diet containing dsRNA to larvae of the lepidopteran species $S$. exigua not only suppressed transcription levels but also led to lethal phenotypes, and proved that SID-1 exists in S. exigua [49]. SID-1 is required for spreading RNA-interfering information between tissues, usually leading to systemic RNAi [50]. In a previous paper, we reported that the oral delivery of an artificial diet containing NITPS dsRNA can induce target gene silencing in $N$. lugens [36]. SID-1 has been found in N. lugens [51], and NlFoxA gene is also expressed in the fat body. Thus, oral delivery of RNA interference can be achieved to knockdown target genes in N. lugens. Further research will address how much dsRNA is required to avoid degradation in the midgut and can reach the target tissues.

\section{Conclusion}

We have demonstrated the existence and characterization of a FoxA gene in $N$. lugens, and have shown that oral ingestion of NlFoxA dsRNA solutions by $N$. lugens nymphs resulted in decreased expression of the target gene, less fecundity and underdeveloped ovarian in the brachypterous adults via regulating vitellogenin expression.

\section{Methods}

\section{Insect rearing}

The $N$. lugens strain was kindly provided by Guangdong Academy of Agricultural Sciences in September 2007 and has been reared in continuous greenhouse laboratory culture conditions since then on rice plants (Huang Hua Zhan, bought from Guangdong Academy of Agricultural Sciences, Guangzhou, China) [36].

\section{Cloning and sequence analysis of NIFoxA}

Total RNA was isolated from four $2^{\text {nd }}$ day brachypterous female adults of $N$. lugens using the Trizol kit (Invitrogen, USA). Its integrity was detected using Agilent 2100 (USA). First-strand cDNA was synthesized with a first strand synthesis kit using reverse transcriptase $\mathrm{X} \mathrm{L}$ (AMV) and an oligo $\mathrm{dT}_{18}$ primer (TaKaRa, Japan). Two pairs of degenerate primers were designed based on the conserved amino acid sequences of fork head from different insects and animals (Table 1). The first-strand cDNA $(1 \mu \mathrm{l})$ was used as a template for PCR using a general protocol. The reaction mixture contained 0.1 
Table 1 Primers used in this study

\begin{tabular}{|c|c|}
\hline Primers & Primer sequence \\
\hline \multicolumn{2}{|c|}{ Degenerate primers } \\
\hline N/FoxAF1 & 5-CACGCSAAGCCBCCVTACTC-3 \\
\hline NIFoxAF2 & 5-ATCACSATGGCBATMCAGAA-3 \\
\hline NIFOXAR1 & 5-TCYTTYTTYTCGTCYTTGAA-3 \\
\hline NIFOXAR2 & 5-CCGTTYTCRAACATGTTVCC-3 \\
\hline \multicolumn{2}{|l|}{ For RACE } \\
\hline 3N/FoxA1 & 5'-AGTTCAATGACTGTTTTGTGAA-3' \\
\hline 3N/FoxA2 & 5'-TTCTGGACSCTGCATCCCGAGT-3' \\
\hline 5N/FoxA1 & 5'-AGAATTCTGGATRGCCATCG-3' \\
\hline 5N/FoxA2 & 5'-GATARGTCTCTGATAGTGTGA-3' \\
\hline \multicolumn{2}{|c|}{ For RT-PCR and real-time PCR } \\
\hline Q NIFoxAF & 5'-TTACACGCACGCTAAACCAC-3' \\
\hline Q NIFOXAR & 5'-CCTCATCAGCCCAAGGGAACAA-3' \\
\hline Q NNgF & 5'-GCATCAATGAACCCAGCTAACTC-3' \\
\hline Q NNgR & 5'-TGGACGGCTCTTTGCATACTCC-3' \\
\hline ACTIN-F & 5'-TGCGTGACATCAAGGAGAAGC-3' \\
\hline$A C T I N-R$ & 5'-CGGCACCTTCACAAAACAG-3' \\
\hline \multicolumn{2}{|c|}{ For NIFoxA dsRNA synthesis } \\
\hline NIFoxA -F & 5'-TTCGAGAACGGCTGCTACCTGCGG-3' \\
\hline NIFoxA -R & 5'-GCTCTCGGCGGGCAGCAGCC-3' \\
\hline \multicolumn{2}{|c|}{ For GFP dsRNA synthesis } \\
\hline GFP-F & 5'-AAGGGCGAGGAGCTGTTCACCG-3' \\
\hline GFP-R & 5'-CAGCAGGACCATGTGATCGCGC-3' \\
\hline \multicolumn{2}{|c|}{ For $N N g$ protein expression } \\
\hline$N N g-E F$ & 5'-GTA CCATGG CCAGTAACT TCCCCAATG TG-3' \\
\hline$N N g-E R$ & 5'-GAT CTCGAGCTTGGCCAAGACAACAACCTTC-3' \\
\hline \multicolumn{2}{|c|}{ For NNg dsRNA synthesis } \\
\hline$N N g-F$ & 5'-ACAGCCAGTCCAACAGCTTCTAC-3' \\
\hline$N N g-R$ & 5'-TGCTGCTGCTGCTGCTGCTTC-3' \\
\hline
\end{tabular}

mM dNTP, $0.5 \mathrm{mM}$ of each degenerate primer and 1.0 $\mathrm{U}$ of HiFi-Taq DNA polymerase (TransGen Biotech, Guangzhou, China) in a total volume of $25 \mu \mathrm{l}$. The first PCR was carried out with the following conditions: initial preheating for $5 \mathrm{~min}$ at $94^{\circ} \mathrm{C}, 35$ cycles at $94^{\circ} \mathrm{C}$ for $30 \mathrm{~s}, 48^{\circ} \mathrm{C}$ for $30 \mathrm{~s}$ and $72^{\circ} \mathrm{C}$ for $1 \mathrm{~min}$, and with a final extension at $72^{\circ} \mathrm{C}$ for $10 \mathrm{~min}$ using the primer pair $N l$ FoxAF1 and NlFoxAR1. The second PCR was performed using another degenerate pair, NlFoxAF2 and $N l$ FoxAR2, with the aforementioned program. The amplified fragment was recovered in a 1\% agarose gel and purified using the Gel Extraction Kit (Omega, USA). Purified DNA was ligated into the pMD18-T vector (TaKaRa, Japan), and recombinant clones were digested with EcoRI and Pst to screen the presence of inserted DNA. Positive clones were sequenced by Invitrogen company (Shanghai, China). To obtain the fulllength NlFoxA cDNA, we used a RACE Kit (CLONTECH, Japan). Specific primers for the 5'- and 3'- Rapid Amplification of cDNA Ends (RACE) were designed based on homologous PCR fragments. The specific primers $5 \mathrm{NlFoxA} 1$ and $5 \mathrm{NlFoxA} 2$ were used for 5 RACE, while $3 N l$ FoxA1 and $3 N l$ FoxA2 were used for 3 'RACE (Table 1). Using the 5'- and 3'-RACE cDNAs as templates, PCR was performed using the $5 \mathrm{NlFoxA} 1$ primer and Universal Primer Mix (UPM, Clontech) by denaturing at $95^{\circ} \mathrm{C}$ for $30 \mathrm{~s}$, followed by 35 cycles of $95^{\circ}$ $\mathrm{C}$ for $30 \mathrm{~s}, 55^{\circ} \mathrm{C}$ for $30 \mathrm{~s}$ and $72^{\circ} \mathrm{C}$ for $2 \mathrm{~min}$, and a final extension at $72^{\circ} \mathrm{C}$ for $10 \mathrm{~min}$. Nested PCR was carried out with the first-round PCR product as a template and the Nested Universal Primer A (NUP, Clontech) and $N l$ FoxA2 primer. The reaction conditions consisted of the followings: $6 \mathrm{~min}$ of initial preheating at $94^{\circ} \mathrm{C}, 30$ cycles of $94^{\circ} \mathrm{C}$ for $30 \mathrm{~s}, 68^{\circ} \mathrm{C}$ for $30 \mathrm{~s}$ and $72^{\circ} \mathrm{C}$ for $40 \mathrm{~s}$, and a final elongation at $72^{\circ} \mathrm{C}$ for $7 \mathrm{~min}$. The RACE products were purified and sequenced as described above. Sequence homologous alignment and similarity searches were carried out by Blast biological software http://www.ncbi.nlm.nih.gov/blast. The signal peptide was analyzed by the SignalP procedure http://www. expasy.ch/SignalP.

\section{Quantitative real-time PCR (RT-qPCR)and Reverse transcriptase PCR (RT-PCR)}

The expression pattern of FoxA in $N$. lugens was detected by RT-qPCR using a Light Cycler 480 system (Roche Diagnostics Indianapolis, IN, USA) and SYBR Premix Ex Taq (Takara, Japan). Briefly, the copy number of the target genes and $C_{T}$ values were negative correlated. It means that one sample containing a higher number of copies of the target gene had a lower $C_{T}$ value. The differences in the $C_{T}$ values of NlFoxA or $\mathrm{NlVg}$ and the corresponding internal control $\beta$-actin $\left(\Delta C_{T}\right)$ were calculated to normalize the difference in the amount of total RNA added to the cDNA reaction mixture and the efficiency of the reverse transcription reaction. The $\Delta C_{\mathrm{T}}$ for the control sample was subtracted from the $\Delta C_{T}$ of the challenged sample. The difference was expressed as a $\Delta \Delta C_{\mathrm{T}}$ value that allowed comparing the expressions of target genes in the challenged sample relative to the control. The expression level of NlFoxA or $N l V g$ were calculated by $2^{-\Delta \Delta C T}$ [52], and the value represented an $\mathrm{n}$-fold difference against the control. All the assays was guided rigidly with MIQE Precis [53].

To investigate the expression pattern of different developmental phases and tissues, we isolated total RNA from the $1^{\text {st }}$ to $5^{\text {th }}$ instar nymphs and from 1 - to 10 day-old brachypterous female adults. The ovary, fat body, midgut, Malpighian tubules, cuticle, and muscle of $3^{\text {rd }}$ brachypterous female adults were carefully collected as described above. The specimen and tissues were rinsed in $1 \times$ PBS buffer several times, and each tissue was pooled from 15-80 individuals. All samples were used for reverse transcription to obtain the first-strand cDNA as above. The primer pairs Q NlFoxAF/Q 
$N l$ FoxAR or Q $N l \mathrm{VgF} / \mathrm{Q} N l \mathrm{VgR}$ (Table 1) were designed to determine the relative expression of $N l F o x A$ or $N l V g$. Each reaction mixture was done in a final volume of 10 $\mu \mathrm{l}$ containing $1 \mu \mathrm{l}$ of the cDNA sample (or standard), $0.2 \mu \mathrm{l}(10 \mathrm{pmoles} / \mu \mathrm{l})$ of each primer and $5 \mu \mathrm{l}$ of SYBR premix Ex Taq. After $10 \mathrm{~s}$ of an initial denaturation at $95^{\circ} \mathrm{C}$, the cycling protocol consisted of 40 cycles of denaturation at $95^{\circ} \mathrm{C}$ for $10 \mathrm{~s}$, annealing at $58^{\circ} \mathrm{C}$ for 20 $\mathrm{s}$, and elongation at $72^{\circ} \mathrm{C}$ for $25 \mathrm{~s}$. A $\beta$-ACTIN (EU179846) cDNA fragment was amplified with ACTIN-F and ACTIN-R primers (Table 1) as an internal control. The quantity of transcripts was estimated from a standard line derived from 10 -fold serial dilutions of cDNA pooled from ten individuals of $2^{\text {nd }}$ brachypterous female adults.

For RT-PCR analysis, amplification was performed using specific primers Q $N l$ FoxAF/Q $N l$ FoxAFR and ACTIN-F/ACTIN-R (Table 1) by denaturing at $95^{\circ} \mathrm{C}$ for $5 \mathrm{~min}$, followed by 28 cycles of $95^{\circ} \mathrm{C}$ for $30 \mathrm{~s}, 58^{\circ} \mathrm{C}$ for $30 \mathrm{~s}$ and $72^{\circ} \mathrm{C}$ for $40 \mathrm{~s}$, with a final extension at $72^{\circ} \mathrm{C}$ for $10 \mathrm{~min}$. Each PCR product $(6 \mu \mathrm{l})$ was electrophoresed and detected by ethidium bromide staining.

\section{$N / \mathrm{g}$ polyclonal antibody generation}

A cDNA fragment containing $N l V g$ partial sequence (4520-5306 bp) was amplified with two primers NlVgEF and NlVg-ER (Table 1), which contain the restriction sites $\mathrm{XhoI}$ and $\mathrm{NcoI}$, respectively. The PCR product was excised with $X h o I$ and $N c o I$, and then subcloned into the pET32a vector (pET-NlVg). The recombinant $N l \mathrm{Vg}$ protein was expressed in BL21 cells induced by IPTG. The E. coli pellet was solubilized in $6 \mathrm{M}$ urea in $50 \mathrm{mM}$ Tri-Cl buffer, pH 8.0, then purified with a Ni-NTA column (GE Healthcare). Purified recombinant NlVg protein was used to immunize rabbits as described previously [54]. The sera of the immunized rabbits were collected as anti-NlVg sera.

\section{RNA interference}

To generate double-stranded RNA (dsRNA), a 432-bp (831-1263 bp) (NlFoxA) and a 600-bp (5252-5852 bp) $(N l V g)$ fragment templates were amplified, as previously described, by PCR using NlFoxA and NlVg cDNAs as templates, with forward and reverse primers containing the T7 primer sequence at the 5 ' ends (Table 1). The amplification reactions protocol comprised 38 cycles of $95^{\circ} \mathrm{C}$ for $35 \mathrm{~s}, 55^{\circ} \mathrm{C}$ for $40 \mathrm{~s}$ and $72^{\circ} \mathrm{C}$ for $60 \mathrm{~s}$, with a final extension step of $72^{\circ} \mathrm{C}$ for $10 \mathrm{~min}$. The sequence was verified by sequencing (Invitrogen company, Shanghai, China). The GFP gene (ACY56286) was used as a control dsRNA. The PCR primers GFP-F and GFP-R were used to amplify the GFP fragment (688 bp) (Table 1 ), and dsRNA corresponding to NlFoxA gene (ds $N l$ FoxA) and $N l \mathrm{Vg}$ gene (ds $N l \mathrm{Vg}$ ) were prepared according to the methods established in our laboratory [55]. The T7 RiboMAX ${ }^{\mathrm{TM}}$ Express RNAi System (Promega, USA) was used for the synthesis. To deliver dsRNA into the body of BPHs, BPHs were reared on an artificial diet [56], with some modifications to the rearing protocol. We used glass cylinders measuring $9.0 \mathrm{~cm}$ in length and $2.0 \mathrm{~cm}$ in diameter as feeding chambers. The dsRNA solution was added to the artificial diet, held between two layers of stretched Parafilm $M$ that were enclosed at the two open ends of the chamber. The diet (each end was loaded $10 \mu \mathrm{L}$ )was renewed every day. The dsRNA concentrations were designated as high dose $(0.5 \mu \mathrm{g} / \mu \mathrm{l})$ and low dose $(0.1 \mu \mathrm{g} / \mu \mathrm{l})$, as previously established [36]. The cylinders were covered with a piece of black cotton cloth, but the two ends where the artificial diet was placed were exposed to light. Insects could feed on the diets by puncturing the inner Parafilm $M$ membrane of the diet pouch. All insects were transferred into chambers and pre-reared on artificial diets for 1 day before initiation of the assays. Then fifteen $3^{\text {rd }}$ instar individuals were transferred into each chamber, and every three chambers were used in each set of triplicate repetitions. The rearing experiments were carried out in a growth cabinet with a humidifier at $27^{\circ} \mathrm{C}$, using $90 \% \mathrm{RH}$ and a $16: 8$-h light:dark photoperiod.

\section{Bioassay and data analysis}

To analyze the influence of NlFoxA and $N l V g$ genes on fecundity of N. lugens, we planted rice plants in small basins $20 \mathrm{~cm}$ deep by $25 \mathrm{~cm}$ in diameter, each covered with two ends of an opened transparent plastic cylinder. The cylinder was $70 \mathrm{~cm}$ in height and $20 \mathrm{~cm}$ in diameter, with two small windows in the wall, leaving a 12$\mathrm{cm}$ gap from the bottom. The top and windows were enclosed with a piece of nylon mesh to prevent the insects from moving in or out. Ten days later, all nymphs were transferred to rice plants that are tillering, the newly emerged brachypterous adults were collected, and each female was matched with one male from each basin of the rice plants. Spawning lasted 15 days, and the parents were removed after oviposition. The offspring were counted by $\mathrm{CO}_{2}$ anesthetization 8 days after the parents were removed.

The efficiency of NlFoxA gene silencing on the effect of knockdown on $N l \mathrm{Vg}$ and fecundity in $\mathrm{BPH}$ were assessed. Experiments employed different concentrations and the same feeding protocol as described above. The brachypterous adults were collected from 1 to 8 days following emergence. Three synchronous larvae were sampled randomly every day in replicates of three. The relative mRNA expression level was analysed by RTqPCR. Primers and reaction conditions were the same as described above. 


\section{Ovary dissection and microscopy}

To determine the impact of gene silencing on the development of ovarioles in $\mathrm{BPH}$, the feeding and raising protocols were the same as described above. The ovaries were dissected from the $3^{\text {rd }}$ day of brachypterous female adult $N$. lugens in $1 \times$ phosphate buffered saline (PBS) $\left(137 \mathrm{mM} \mathrm{NaCl} ; 2.68 \mathrm{mM} \mathrm{KCl} ; 1.47 \mathrm{mM} \mathrm{KH}_{2} \mathrm{PO}_{4}\right.$; and $8.10 \mathrm{mM} \mathrm{Na}_{2} \mathrm{HPO} 4, \mathrm{pH} 7.0$ ), followed by fixation in $3.8 \%$ formaldehyde in $1 \times$ PBS for 20 minutes at room temperature. Dissected ovaries were washed three times for 10 minutes with $0.2 \%$ Triton-X 100 (Sigma, USA) in $1 \times$ PBS. After washing, ovaries were photographed with a Leica DMR connected to a Fuji FinePix S2 Pro digital camera(Germanny).

\section{Enzyme-linked immunosorbent assay (ELISA) and western blot analysis}

Variations of $\mathrm{Vg}$ protein caused by RNAi were determined through ELISA and western blot analysis. Total proteins were extracted from fifteen individuals' ovaries of BPH in four different treatment groups. The same total amount of protein was coated on microtiter plates. Briefly, these total proteins were diluted to $0.5 \mathrm{mg} / \mathrm{mL}$ with carbonate buffered solution ( $0.05 \mathrm{M}, \mathrm{pH}$ 9.6) and used to coat 96-well microplates at $100 \mu \mathrm{L} /$ well. Following overnight incubation at $4^{\circ} \mathrm{C}$, the plates were washed three times with PBS$\mathrm{T}$ (PBS containing $0.05 \%$ Tween 20, USA) and blocked with $5 \%$ skim milk in PBS $(100 \mu \mathrm{L} /$ well $)$. After incubation at $37^{\circ} \mathrm{C}$ for $1 \mathrm{~h}$, the plates were washed 3 times with PBST. The rabbit polyclonal antibodies were serially diluted through the wells using PBS, and the plates were incubated for $1 \mathrm{~h}$ at $37^{\circ} \mathrm{C}$. After a subsequent wash step, an enzyme-labeled second antibody (goat-anti-rabbit labeled with horse radish peroxidase, GAM-HRP (BOSTER, Wuhan, China) was diluted 1:8000 with PBST and added to the plates $(100 \mu \mathrm{L} /$ well). The plates were incubated for $1 \mathrm{~h}$ at $37^{\circ} \mathrm{C}$ and washed 4 times with PBST $(200 \mu \mathrm{L} /$ well). The substrate solution (TMB buffer, $\mathrm{pH}$ 5.5) was then added at $100 \mu \mathrm{L} /$ well to each well. After $10 \mathrm{~min}$ at room temperature, the reaction was terminated by $50 \mu \mathrm{L} /$ well 2 $\mathrm{M} \mathrm{H}_{2} \mathrm{SO}_{4}$. The absorbance was measured at $450 \mathrm{~nm}$. PBST (0.1 M, pH 7.4) instead of polyclonal antibodies was used in the blank control. The value of $\mathrm{OD}_{450}$ indicates the concentration of $N I V g$ protein.

Western-blotting analysis were modified according to the methods from the previously described [57]. Briefly, totally $300 \mu$ g ovaries proteins (as above) were separated on a $12 \%$ SDS-PAGE gel, transferred to NT membranes (PVDF, Bio-Rad), immunoblotted with anti-Vg serum (diluted 1:5000) and an IgG goat anti-rabbit antibody conjugated with HRP was used for secondary antibody (BOSTER, Wuhan, China, 1:5000 dilution), finally visualized by ECL (enhanced chemiluminescence).

\section{Statistical analysis}

The results are expressed as the means ( \pm S.E.M.). SPSS 13.0 software was used to perform $t$-tests to identify significant differences at a 95\% confidence level $(\mathrm{p}<0.05)$.

\section{Acknowledgements}

We are grateful to Dr. Yang Zhang (Guangdong Academy of Agricultural Sciences, China) for kindly supplying the N. lugens strain and to Dr. XiaoQiang Yu (University of Missouri-Kansas City, USA) for his suggestions. This work was supported by the National Natural Science Foundation of China (30930061) and National Basic Research Program of China (2010CB126200).

\section{Authors' contributions}

XD has completed most of the experiment and proofread, developed the concept and wrote the manuscript. YZ has reared the PBH and complete partial experiment. JZ, ZS, JC and JC were involved in the data and sequence analysis. WZ has designed the experiment and polished the manuscript. All the authors have approved the final form of the manuscript.

Received: 5 September 2011 Accepted: 31 December 2011

Published: 31 December 2011

\section{References}

1. Manjunath TM: A note on oviposition in the maeropterous and braehypterous forms of the rice brown planthopper, Nilaparvata lugens Stal (Homoptera, Delphacidae). Proc Indian Acad Sci 1977, 86 B(6):405-408.

2. Yu D: biological characteristics and identify in Brown plant hopper Nilaparvata lugens . agriculture technique service 2007, 24(2):45-46.

3. The study of anther culture in 'three lines' breeding and utilization of heterosis in Oryza sativa Subsp. hsien. Proc. Symp. Edited by: Ling T, Huang H, Liang C, Chun P. Peking: Science Press; 1978:

4. Sogawa K: Feeding of the rice plant and leaf hoppers. Revplant Prot Res 1973, 6:31-43.

5. Otake A: Population characteristics of the brown planthopper,Lilaparbata lugens (Hemiptera: Delphacidae), with special reference to difference in Japan and the tropics. J Appl Ecol 1978, 15(385-394).

6. Hibino H: Biology and epidemiology of rice viruses. Annu Rev Phytopathol 1996, 34:249-274.

7. Genetics of and breding resistance to the brown planthopper. Edited by: Khush G. Philippines.: IRRI, Los Banos; 1979:.

8. Wang M: outbreak status, current prevention and sustainable management technique of Nilaparvata lugens. Journal of Anhui Agricultural Sciences 2007, 35:2944-2945.

9. Liu Z, Han Z: Fitness costs of laboratory-selected imidacloprid resistance in the brown planthopper, Nilaparvata lugens Stal. Pest Manag Sci 2006, 62(3):279-282.

10. Impact of insecticides on the resistance and resurgence of rice planthoppers. Edited by: Heinrichs E. New York: Chapman 1994:.

11. Vontas JG, Small GJ, Hemingway J: Comparison of esterase gene amplification, gene expression and esterase activity in insecticide susceptible and resistant strains of the brown planthopper, Nilaparvata lugens (Stal). Insect Mol Biol 2000, 9(6):655-660.

12. Wu J, Xu J, Yuan S, Liu J, Jian Y, Xu J: Pesticide-induced susceptibility of rice to brown planthopper Nilaparvata lugens. Entomol Exp Appl 2001, 100:119-126.

13. Klowden MJ: Physiological systems in insects. Amsterdam; Boston: Elsevier/Academic Press;i, 2007.

14. Chen J, Cheng S, Yang L, Ying S: The ovarial development of the brown planthopper (Nilaparvata lugens stal) and it's relation to migration. Acta Entomologica Sinica 1979, 22(3):280-287.

15. Kaestner KH, Knochel W, Martinez DE: Unified nomenclature for the winged helix/forkhead transcription factors. Genes Dev 2000, 14(2):142-146.

16. Kaufmann E, Knochel W: Five years on the wings of fork head. Mech Dev 1996, 57(1):3-20

17. Weigel $D$, Jürgens $G$, Küttner $F$, Seifert $E$, Jäckle $H$ : The homeotic gene fork head encodes a nuclear protein and is expressed in the terminal regions of the Drosophila embryo. Cell 1989, 57(4):645-658. 
18. Carlsson P, Mahlapuu M: Forkhead transcription factors: key players in development and metabolism. Dev Biol 2002, 250(1):1-23.

19. Birkenkamp KU, Coffer PJ: FOXO transcription factors as regulators of immune homeostasis: Molecules to die for? Journal of Immunology 2003 171(4):1623-1629.

20. Su N, Thiaville MM, Awad K, Gjymishka A, Brant JO, Yang TP, Kilberg MS: Protein or Amino Acid Deprivation Differentially Regulates the Hepatic Forkhead Box Protein A (FOXA) Genes Through an Activating Transcription Factor-4-Independent Pathway. Hepatology 2009, 50(1):282-290.

21. Kaestner $\mathrm{KH}$ : The FoxA factors in organogenesis and differentiation. Curr Opin Genet Dev 2010, 20(5):527-532

22. Sim C, Denlinger DL: Insulin signaling and FOXO regulate the overwintering diapause of the mosquito Culex pipiens. Proc Natl Acad Sci USA 2008, 105(18):6777-6781.

23. Bao B, Hong B, Feng Q- L, Xu W-H: Transcription factor fork head regulates the promoter of diapause hormone gene in the cotton bollworm, Helicoverpa armigera, and the modification of SUMOylation. Insect Biochemistry and Molecular Biology 2011, 41(9):670-679.

24. Hong B, Zhang ZF, Tang SM, Yi YZ, Zhang TY, Xu WH: Protein-DNA interactions in the promoter region of the gene encoding diapause hormone and pheromone biosynthesis activating neuropeptide of the cotton bollworm, Helicoverpa armigera. Biochim Biophys Acta 2006, 1759(3-4):177-185.

25. Thompson DM, Khalil SM, Jeffers LA, Ananthapadmanaban U, Sonenshine DE, Mitchell RD, Osgood CJ, Apperson CS, Michael Roe R: In vivo role of 20-hydroxyecdysone in the regulation of the vitellogenin mRNA and egg development in the American dog tick, Dermacentor variabilis (Say). J Insect Physiol 2005, 51(10):1105-1116.

26. Sirotkin AV: Transcription factors and ovarian functions. J Cell Physiol 2010, 225(1):20-26.

27. Hansen IA, Sieglaff DH, Munro JB, Shiao SH, Cruz J, Lee IW, Heraty JM, Raikhel AS: Forkhead transcription factors regulate mosquito reproduction. Insect Biochem Mol Biol 2007, 37(9):985-997.

28. Fire A, Xu S, Montgomery MK, Kostas SA, Driver SE, Mello CC: Potent and specific genetic interference by double-stranded RNA in Caenorhabditis elegans. Nature 1998, 391(6669):806-811.

29. Sirotkin AV: RNA interference and ovarian functions. J Cell Physiol 2010, 225(2):354-363.

30. Roberts J, Baum JA, Bogaert T, Clinton W, Heck GR, Feldmann P, Ilagan O, Johnson S, Plaetinck G, Munyikwa T, et al: Control of coleopteran insect pests through RNA interference. Nat Biotechnol 2007, 25(11):1322-1326.

31. Zhang W, Chen X, Tian H, Zou L, Tang B, Hu J: Disruption of Spodoptera exigua larval development by silencing chitin synthase gene $A$ with RNA interference. B Entomol Res 2008, 98(6):613-619.

32. Eaton $\mathrm{BA}$, Fetter $\mathrm{RD}$, Davis $\mathrm{GW}$ : Dynactin is necessary for synapse stabilization. Neuron 2002, 34(5):729-741.

33. Chen XY, Mao YB, Cai WJ, Wang JW, Hong GJ, Tao XY, Wang LJ, Huang YP: Silencing a cotton bollworm P450 monooxygenase gene by plantmediated RNAi impairs larval tolerance of gossypol. Nat Biotechnol 2007, 25(11):1307-1313.

34. Tian $\mathrm{HG}$, Peng $\mathrm{H}$, Yao Q, Chen $\mathrm{HX}$, Xie Q, Tang B, Zhang WQ: Developmental Control of a Lepidopteran Pest Spodoptera exigua by Ingestion of Bacteria Expressing dsRNA of a Non-Midgut Gene. Plos One 2009, 4(7)

35. Timmons L, Court DL, Fire A: Ingestion of bacterially expressed dsRNAs can produce specific and potent genetic interference in Caenorhabditis elegans. Gene 2001, 263(1-2):103-112.

36. Chen J, Zhang D, Yao Q, Zhang J, Dong X, Tian H, Zhang W: Feedingbased RNA interference of a trehalose phosphate synthase gene in the brown planthopper, Nilaparvata lugens. Insect Mol Biol 2010, 19(6):777-786.

37. Schroder R, Eckert C, Wolff C, Tautz D: Conserved and divergent aspects of terminal patterning in the beetle Tribolium castaneum. Proc Natl Acad Sci USA 2000, 97(12):6591-6596.

38. Huo Z, Chen L, Ye C, Liu L: Effect of climate on outbreak of China rice planthopper. Journal of Natural Disasters 2002, 11:97-102.

39. Awmack CS, Leather SR: Host plant quality and fecundity in herbivorous insects. Annu Rev Entomol 2002, 47:817-844.
40. Verlinden H, Badisco L, Marchal E, Van Wielendaele P, Vanden Broeck J: Endocrinology of reproduction and phase transition in locusts. Gen Comp Endocrinol 2009, 162(1):79-92.

41. de-Leon SB: The conserved role and divergent regulation of foxa, a paneumetazoan developmental regulatory gene. Dev Biol 2010.

42. Julien E, Bordeaux MC, Garel A, Couble P: Fork head alternative binding drives stage-specific gene expression in the silk gland of Bombyx mori. Insect Biochemistry and Molecular Biology 2002, 32(4):377-387.

43. Cao C, Liu Y, Lehmann M: Fork head controls the timing and tissue selectivity of steroid-induced developmental cell death. J Cell Biol 2007, 176(6):843-852.

44. Friedman JR, Kaestner $\mathrm{KH}$ : The Foxa family of transcription factors in development and metabolism. Cell Mol Life Sci 2006, 63(19-20):2317-2328.

45. Hu DG, Mackenzie PI: Forkhead box protein A1 regulates UDPglucuronosyltransferase 2B15 gene transcription in LNCaP prostate cancer cells. Drug Metab Dispos 2010, 38(12):2105-2109.

46. Clements AN: The Biology of Mosquitoes. Chapman \& Hall, London 1992.

47. Mitchell lii RD, Ross E, Osgood C, Sonenshine DE, Donohue KV, Khalil SM, Thompson DM, Michael Roe R: Molecular characterization, tissue-specific expression and RNAi knockdown of the first vitellogenin receptor from a tick. Insect Biochemistry and Molecular Biology 2007, 37(4):375-388.

48. Kokoza VA, Martin D, Mienaltowski MJ, Ahmed A, Morton CM, Raikhel AS: Transcriptional regulation of the mosquito vitellogenin gene via a blood meal-triggered cascade. Gene 2001, 274(1-2):47-65.

49. Tian H, Peng H, Yao Q, Chen H, Xie Q, Tang B, Zhang W: Developmental control of a lepidopteran pest Spodoptera exigua by ingestion of bacteria expressing dsRNA of a non-midgut gene. Plos One 2009, 4(7): e6225.

50. Winston WM, Molodowitch C, Hunter CP: Systemic RNAi in C. elegans requires the putative transmembrane protein SID-1. Science 2002, 295(5564):2456-2459.

51. Zha WJ, Peng XX, Chen RZ, Du B, Zhu LL, He GC: Knockdown of Midgut Genes by dsRNA-Transgenic Plant-Mediated RNA Interference in the Hemipteran Insect Nilaparvata lugens. Plos One 2011, 6(5)

52. Livak KJ, Schmittgen TD: Analysis of relative gene expression data using real-time quantitative PCR and the 2(-Delta Delta C(T)) Method. Methods 2001, 25(4):402-408.

53. Bustin SA, Beaulieu JF, Huggett J, Jaggi R, Kibenge FS, Olsvik PA, Penning LC, Toegel S: MIQE precis: Practical implementation of minimum standard guidelines for fluorescence-based quantitative real-time PCR experiments. BMC Mol Biol 2010, 11:74

54. Cui SY, Xu WH: Molecular characterization and functional distribution of $\mathrm{N}$-ethylmaleimide-sensitive factor in Helicoverpa armigera. Peptides 2006, 27(6):1226-1234

55. Chen X, Tian H, Zou L, Tang B, Hu J, Zhang W: Disruption of Spodoptera exigua larval development by silencing chitin synthase gene A with RNA interference. Bull Entomol Res 2008, 98(6):613-619.

56. Fu Q, Zhang Z, Hu C, Lai F, Sun Z: A chemically defined diet enables continuous rearing of the brown planthopper, Nilaparvata lugens (Stål) (Homoptera: Delphacidae). Appl Entomol Zool 2001, 36(1):111-116.

57. Mitsumasu K, Azuma M, Niimi T, Yamashita O, Yaginuma T: Changes in the expression of soluble and integral-membrane trehalases in the midgut during metamorphosis in Bombyx mori. Zoolog Sci 2008, 25(7):693-698.

doi:10.1186/1471-2199-12-53

Cite this article as: Dong et al:: Fork head transcription factor is required for ovarian mature in the brown planthopper, Nilaparvata lugens (Stål). BMC Molecular Biology 2011 12:53. 